\title{
Immunohistochemical evaluation of microphthalmia-associated transcription factor expression in giant cell lesions
}

\author{
Raja R Seethala ${ }^{1}$, John R Goldblum² ${ }^{2}$, David G Hicks², Micheal Lehman², Jasvir S Khurana ${ }^{3}$, \\ Terry L Pasha ${ }^{1}$ and Paul J Zhang ${ }^{1}$ \\ ${ }^{1}$ University of Pennsylvania Medical Center, Philadelphia, PA, USA; ${ }^{2}$ Cleveland Clinic Foundation, \\ Cleveland, OH, USA and ${ }^{3}$ Temple University, Philadelphia, PA, USA
}

\begin{abstract}
Microphthalmia-associated transcription factor (Mitf), a member of the helix-loop-helix transcription factor subfamily, normally expressed in mononuclear and multinucleated osteoclasts, is involved in the terminal differentiation of osteoclasts. Dysfunction of osteoclast activity resulting from abnormal Mitf expression has been implicated in osteopetrosis. Numerous other giant cells of various types including osteoclast-like giant cells seen in various tumors, traditionally thought to be monocyte derived, are seen in a variety of bone and extraosseous lesions. Using a monoclonal antibody with a standard immunohistochemical technique on paraffin sections, we evaluated expression of Mitf in $\mathbf{8 9}$ various giant cell lesions including giant cell tumor of bone (n26), giant cell tumor of tendon sheath/pigmented villonodular synovitis (n24), giant cell reparative granuloma (n3), aneurysmal bone cysts (n11), chondroblastomas (n7), foreign body giant cell reaction (n10), and sarcoidosis (n8). We also evaluated three cases of osteopetrosis and 27 various tissues without monocytederived giant cells (nine bone marrows, nine products of conception, seven lymph nodes with sinus histiocytosis, one granulation tissue and one thymus). Nuclear Mitf immunoreactivity was evaluated. Mitf was variably expressed in the monocyte-derived giant cells and/or the adjacent mononuclear cells/histiocytes in 23 $(89 \%)$ giant cell tumors of the bone, $23(96 \%)$ giant cell tumors of tendon sheath/pigmented villonodular synovitis, three $(100 \%)$ giant cell reparative granuloma, eight $(73 \%)$ aneurysmal bone cysts, five $(71 \%)$ chondroblastomas, eight $(80 \%)$ foreign-body giant cell reactions, and six $(75 \%)$ sarcoidoses. No Mitf immunoreactivity was detected in cases of osteopetrosis and giant cells of nonmonocyte origin. Mitf immunoreactivity is rare in tissues with rich mononuclear cells/histiocytes but no monocyte derived giant cells. These findings support the notion that giant cells in giant cell lesions are likely derived from adjacent mononuclear cells and Mitf might play a role in the multinucleation process of such cells.

Modern Pathology (2004) 17, 1491-1496, advance online publication, 18 June 2004; doi:10.1038/modpathol.3800211
\end{abstract}

Keywords: microphthalmia; Mitf; osteoclasts; osteoclast-like giant cells; giant cell tumor; giant cell reaction; mononuclear cells; immunohistochemistry

Microphthalmia-associated transcription factor (Mitf) belongs to the beta helix-loop-helix transcription factor family (bHLH) and is integral to the differentiation of melanocytes, mast cells, natural killer cells and osteoclasts. ${ }^{1-4}$ Mitf expression is stimulated via a receptor activator of NF- $\kappa$-B (RANKL). ${ }^{5,6}$ Mitf transactivates the promoters of genes such as cathepsin- $\mathrm{K}^{7}$ when it forms a homodimer or a heterodimer with other bHLH proteins such as Tfe3, Tfeb, Tfec. ${ }^{2}$ Mitf appears to regulate

Correspondence: Dr RR Seethala, MD, 6 Founders, Department of Pathology, 3400 Spruce Street, Philadelphia, PA 19104, USA.

E-mail: seethalr@uphs.upenn.edu

Received 6 October 2003; revised 18 March 2004; accepted 13 May 2004; published online 18 June 2004 the expression of a variety of proteins such as tartrate-resistant acid phosphatase (TRAP) and cathepsin $\mathrm{K}^{3,7,8}$ At least six isoforms (A, B, C, D, $\mathrm{H}$, and $\mathrm{M}$ ) of Mitf have been identified to date, each of which contains a unique $\mathrm{N}$-terminal domain as well as a common domain B1b. ${ }^{1}$ Each of these isoforms is variably expressed in the different cell lines with Mitf-D preferentially expressed in osteoclasts and osteoclast progenitors. ${ }^{1}$ Mutations in Mitf have been implicated in numerous disease phenotypes, including osteopetrosis., ${ }^{2,7,9}$ To date, over 20 mutations have been identified, and the majority of these appear to be inherited in a semidominant fashion. ${ }^{2}$

Multinucleated giant cells such as Langhans giant cells, foreign-body giant cells, and the so-called 
osteoclast-like giant cells share morphologic similarities with the giant cell form of osteoclasts in bone are characteristically seen in many bony and extraosseous lesions including granulomatous lesions such as sarcoidosis, foreign-body giant cell reaction, and giant cell tumors of the bone and tendon sheath (Figure 1). Although morphologic and ultrastructural differences exist between osteoclasts and these other giant cell types, in general, both are thought to arise from monocytes via a multinucleation process as a result of the appropriate milieu of extracellular debris and
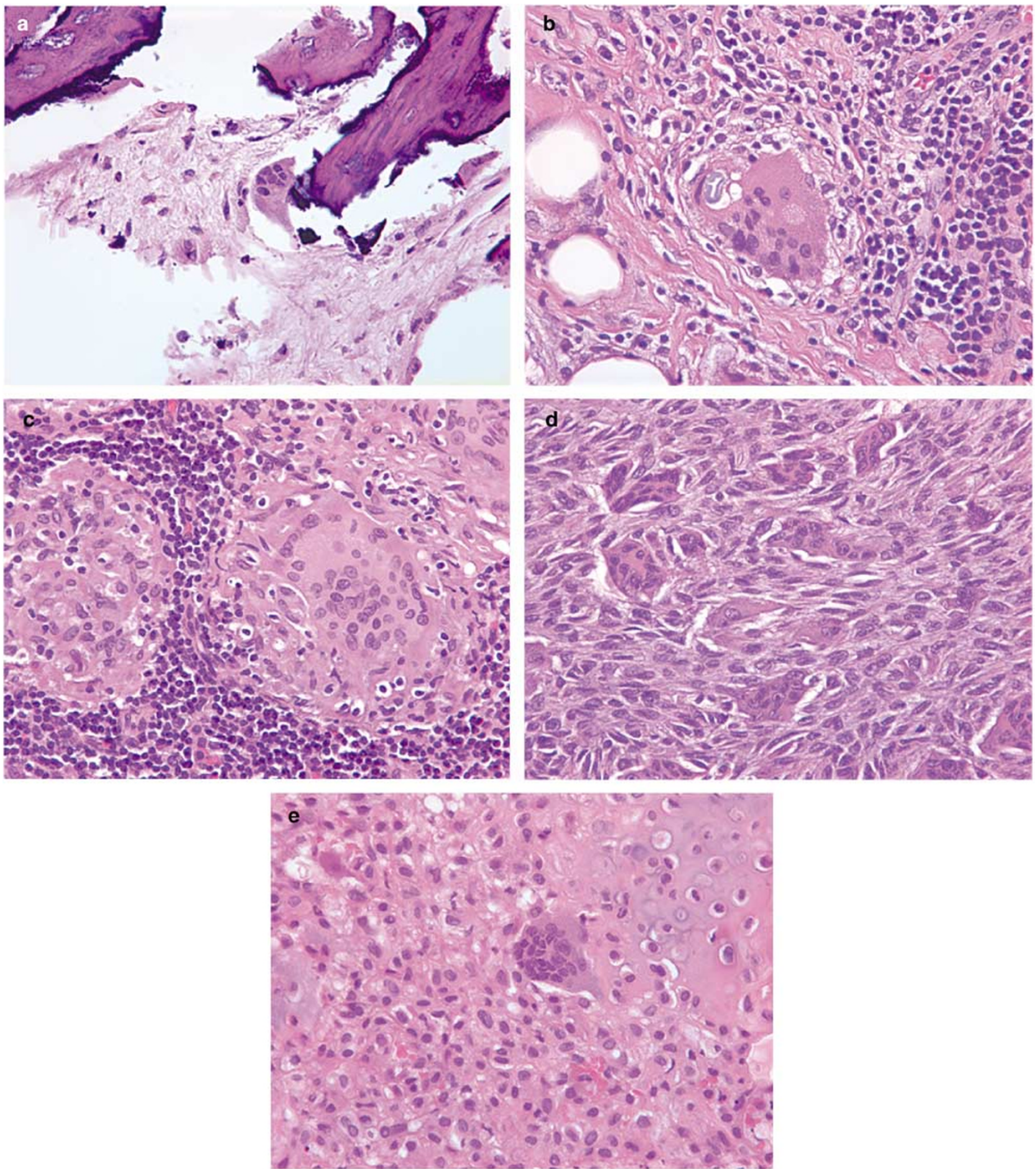

Figure 1 The histomorphologic similarities on hematoxylin and eosin (H\&E) staining of various monocyte-derived giant cells: (a) a few rare osteoclasts identified in osteopetrosis $(\times 40)$, (b) foreign-body giant cell reaction with suture material $(\times 40)$, (c) giant cells in sarcoidosis $(\times 40)$, $(\mathbf{d})$ giant cell tumor of the bone $(\times 40)$, and $(\mathbf{e})$ chondroblastoma with osteoclast-like giant cells $(\times 40)$. 
cytokines. ${ }^{10,11}$ Many functional surface markers such as $\mathrm{Na}+/ \mathrm{K}+$ ATPase, $\mathrm{H}+$ ATPase, calcitonin receptor, and TRAP are known to be expressed in both osteoclasts and monocyte-derived giant cells. ${ }^{11}$ Additionally, in vitro studies have shown increased expression of certain isoforms of $\mathrm{Na}+/ \mathrm{K}+$ ATPase, as well as calcitonin receptor in osteoclasts during the multinucleation process. ${ }^{10,11}$ However, transcription factor expression such as that of Mitf has not been well characterized in monocyte-derived giant cells other than osteoclasts.

\section{Materials and methods}

A series of 89 monocyte-derived giant-cell-rich lesions were randomly retrieved from the surgical pathology files of both the University of Pennsylvania Medical Center and Cleveland Clinic Foundation for Mitf immunoevaluation. There were 26 giant cell tumors of bone, 24 giant cell tumors of tendon sheath/pigmented villonodular synovitis, three giant cell reparative granulomas, 11 aneurysmal bone cysts, seven chondroblastomas, 10 cases of foreign-body giant cell reaction, and eight cases of sarcoidosis (Figure 1). In addition, Mitf was also evaluated in 30 lesions and tissues without monocyte-derived giant cells: three cases of osteopetrosis, nine samples of non-neoplastic bone marrow, nine samples of products of conception, seven samples of lymph nodes with sinus histiocytosis, one sample of granulation tissue, and one sample of normal thymus. All tissues were fixed in $10 \%$ formalin. Tissues of the bony lesions were also subjected to routine standard decalcification before embedding. In all, $4 \mu \mathrm{m}$ thick sections were cut from the paraffin blocks and used for immunohistochemical staining. The sections were pretreated by boiling with $1 \times$ EDTA for $20 \mathrm{~min}$ at $100^{\circ} \mathrm{C}$. Sections were then incubated with primary antibody for $60 \mathrm{~min}$ at room temperature. A nonisoform-specific monoclonal antibody to Mitf (D5, 1:100, 1:50 for decalcified tissues, NeoMarkers/Labvision) was used for immunohistochemical detection with Envision plus (DAKO) on a DAKO autostainer. A formalin-fixed, paraffinembedded melanoma tissue was used for positive control. The primary antibody was substituted by 1:1000 normal mouse serum on an additional section of each case as negative control. Mitf nuclear reactivity was evaluated by light microscopy and when present, the percentage of positive nuclei was estimated separately in the mononuclear and multinucleated cells.

\section{Results}

Nuclear Mitf expression was variably detected in 76 of $89(85 \%)$ cases with monocyte-derived giant cells (Figure 2a-c). Mitf reactivity was found in the giant cells in 23 of $26(88 \%)$ cases of giant cell tumor of bone, 23 of $24(96 \%)$ cases of giant cell tumor of tendon sheath/pigmented villonodular synovitis, three of three $(100 \%)$ cases of giant cell reparative granulomas, eight of $11(73 \%)$ aneurysmal bone cysts, five of seven $(71 \%)$ chondroblastomas, six of eight $(75 \%)$ cases of sarcoidosis, and eight of 10 $(80 \%)$ cases of foreign-body giant cell reaction. The mean percentage of Mitf positivity in monocytederived giant cells in these cases ranged from 23 to $78 \%$. Variable nuclear Mitf expression was also detected in a subset of histiocytic mononuclear cells usually found adjacent to the Mitf-positive monocyte-derived giant cells in all but one of the cases containing Mitf-positive monocyte-derived giant cells. In giant cell tumors of the tendon sheath/ pigmented villonodular synovitis, both mononuclear histiocytes as well as some of the so-called synoviocyte-like small round cells were Mitf positive. Similarly, the mononuclear cells that were Mitf positive in giant cell tumors of the bone are comprised of histiocytic mononuclear cells adjacent to giant cells as well as the small round stromal mononuclear cells (Figure 2b). In chondroblastomas, however, the rare mononuclear cells that were Mitf positive were always histiocytic mononuclear cells rather than lesional chondroblasts (Figure 2c). In cases with Mitf-negative monocyte-derived giant cells, none of the adjacent mononuclear cells were Mitf positive. The range of mean percentage of Mitf positivity in mononuclear cells was $6-60 \%$.

In tissues with numerous histiocytic mononuclear cells but no monocyte-derived giant cells, Mitf was only rarely detected (mean percentage $4 \%$ ) in five of the seven lymph nodes with sinus histiocytosis but not in one thymus and one granulation tissue. Mitf expression was not detected in either osteoclasts or mononuclear osteoclast precursors in three cases of osteopetrosis, nor in any giant cells of nonmonocyte origin such as megakaryocytes in nine bone marrow specimens and syncytiotrophoblasts in nine products of conception (Figure $2 \mathrm{~d}$ and e). The results are summarized in Table 1.

\section{Discussion}

Both osteoclasts and other monocyte-derived giant cells have similar morphology and share similar roles in the resorption or phagocytosis of extracellular materials. ${ }^{11}$ These cells share many surface proteins such as $\mathrm{Na}+/ \mathrm{K}+$ ATPase, $\mathrm{H}+$ ATPase, calcitonin receptor, and TRAP, which are important to their cellular function. ${ }^{11}$ Mitf expression has been speculated to upregulate these surface proteins, particularly at the time of multinucleation in osteoclasts. Given the morphologic similarities between osteoclasts and other monocyte-derived giant cells, one might expect Mitf expression in the latter. ${ }^{11}$

In this study, utilizing immunohistochemical methods, we have shown that Mitf was indeed expressed in the other monocyte-derived giant cells in various inflammatory and neoplastic conditions 

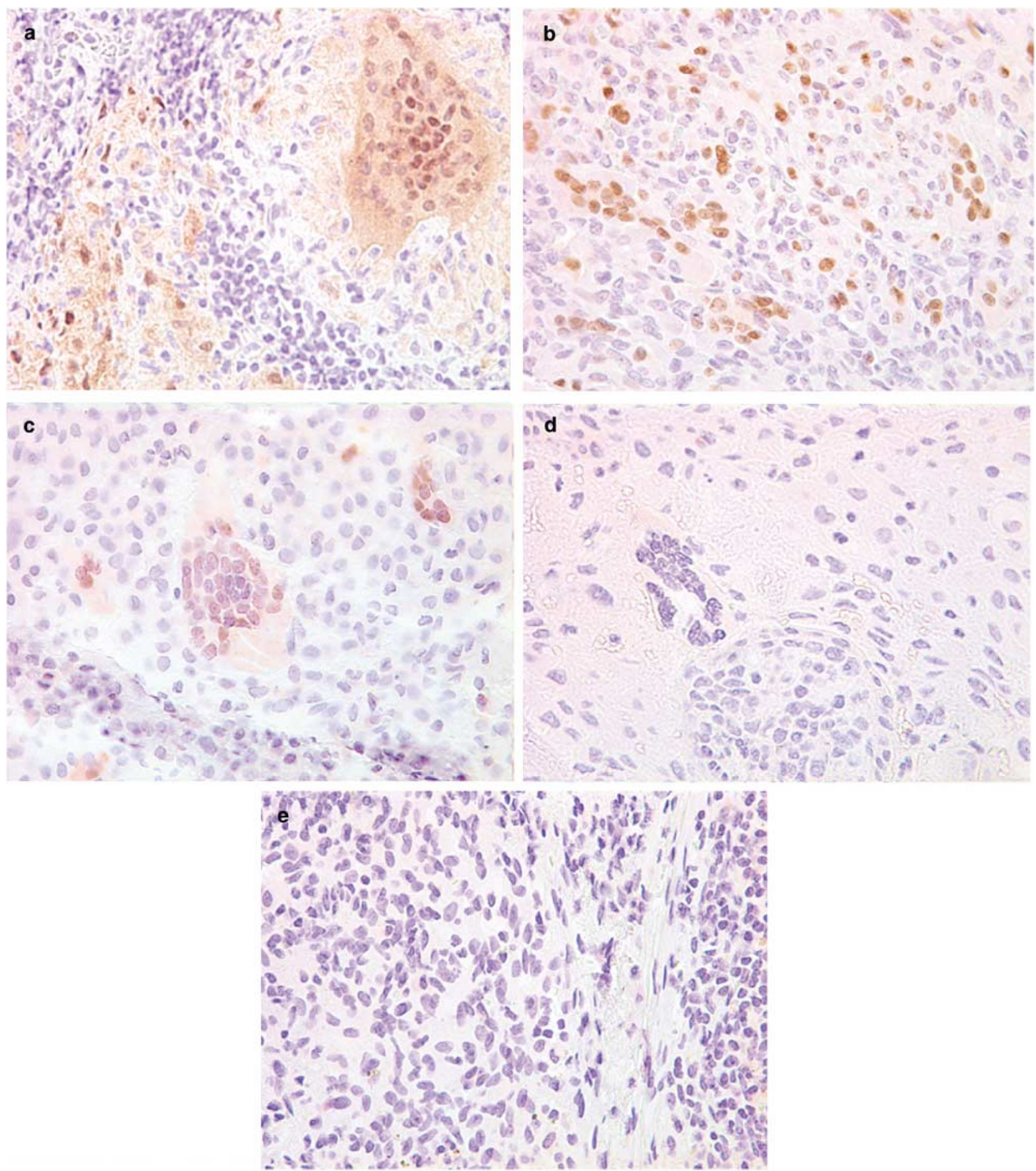

Figure 2 Mitf staining is positive in monocyte-derived giant cells and scattered mononuclear cells in (a) sarcoidosis $(\times 40)$, (b) giant cell tumor of the bone $(\times 40)$, and $(\mathbf{c})$ chondroblastoma $(\times 40)$, while negative in reactive histiocytes or MNC in (d) non-OLGC such as syncytiotrophoblasts in products of conception $(\times 40)$ and $(\mathbf{e})$ sinus histiocytosis of the lymph node $(\times 40)$.

as well as a subset of mononuclear cells adjacent to the monocyte-derived giant cells, but rare in those unassociated with monocyte-derived giant cells. Our study also demonstrated that Mitf was not expressed in nonmonocyte-derived giant cells such as megakaryocytes and syncytiotrophoblasts. Since Mitf expression is restricted in our study to the monocyte-derived giant cells and the adjacent mononuclear cells, which are likely, then, the precursors to these giant cells. 
Multinucleation of mononuclear cells is a complex process. It is still unclear whether the multinucleation process is due to an incomplete divided mitotic process or fusion of the mononuclear cells. ${ }^{10,11}$ More recent evidence has shown that multinucleation requires a certain microenvironment such as a bony surface or a chronic inflammatory site, an optimal cell-cell contact between osteoclasts/other mononuclear precursors and certain local growth factors such as IL-1, M-CSF, interferon- $\gamma$, osteoprotegerin ligand, and 1,25 $\alpha(\mathrm{OH})_{2} \mathrm{D}_{3} \cdot{ }^{11,12}$ Fusion of osteoclasts and mononuclear precursors appears to be mediated by surface proteins such as MFR, CD44, and CD47. ${ }^{11,12}$ The fact that Mitf nuclear expression was frequently detected in monocyte-derived giant cells and the adjacent mononuclear cells but only rarely in the mononuclear cells unassociated with formation of monocyte-derived giant cells suggests involvement of Mitf in the multinucleation process. However, the exact role of Mitf in the multinucleation process has yet to be elucidated. Possible mechanisms include interactions between the above cell-cell adhesion molecules and growth factors known to regulate the multinucleation process and terminal differentiation of the mononuclear cells, and/or transactivation of other growth factors or pathways regulating this process.

Abnormal Mitf expression in osteoclasts, whether quantitative or qualitative, has been associated with various osteopetrosis phenotypes, which result from defects in bone resorption. It is known that abnormalities of Mitf expression may also lead to defective mononuclear/histiocytic function and multinucleation in osteoclasts in some forms of osteopetrosis and compromise their role in host immunosurveillance, which contributes to the increased frequency of osteomyelitis seen in patients with osteopetrosis. ${ }^{13}$ Since multinucleation of mononuclear cells is commonly seen in inflammatory and reactive conditions, defects in Mitf expression might compromise the role of these extraosseous mononuclear cells in host defense.

The histogenesis and the neoplastic nature of giant cell tumor of bone and giant cell tumors of the tendon sheath are still debated. ${ }^{14-18}$ Initially, the association of giant cell tumors of bone and trauma, and the reproducibility of lesions by intra-articular injection with blood led to the notion that this was a reactive process. ${ }^{18}$ More recently, cell culture, cytogenetic and immunohistochemical analyses have favored a neoplastic process, with a neoplastic mononuclear cell component and a reactive giant cell component. ${ }^{16,17}$ The finding of Mitf reactivity in both the osteoclast-like giant cells and the small, round to ovoid mononuclear cells in giant cell tumor of bone and giant cell tumors of the tendon sheath are consistent with previous studies suggesting that both these cell types likely derive from the same lineage as that of normal osteoclasts in bone. ${ }^{15,18}$ The expression of Mitf in the 
osteoclast-like giant cells and the adjacent neoplastic mononuclear cells raises the possibility that the giant cells in these cases are part of the neoplastic process, although more extensive immunophenotypic and molecular analysis would be necessary to define the relationship between the mononuclear cells and the giant cells. In contrast, none of the lesional chondroblasts in chondroblastoma were Mitf positive confirming that the osteoclast-like giant cells in these lesions are likely not part of the neoplastic process.

In summary, our findings suggest that monocytederived giant cells in all giant cell lesions are likely derived from adjacent mononuclear histiocytic precursors and Mitf might play a role in the histogenesis of such cells. Owing to their similarities in morphology and Mitf expression pattern, the cellular elements of all these giant cell lesions, neoplastic or reactive, intraosseous or extraosseous, are related and likely share the same precursor. Although traditionally monocyte-derived giant cells have been considered non-neoplastic, they may be derived from the neoplastic mononuclear cell element in giant cell tumor of bone.

\section{References}

1 Takeda K, Yasumoto K, Kawaguchi N, et al. Mitf-D, a newly identified isoform, expressed in the retinal pigment epithelium and monocyte-lineage cells affected by Mitf mutations. Biochim Biophys Acta 2002;1574:15-23.

2 Steingrimsson E, Tassarollo L, Pathak B, et al. Mitf and Tfe3, two members of the Mitf-Tfe family of bHLH-Zip transcription factors, have important but functionally redundant roles in osteoclast development. Proc Natl Acad Sci USA 2002;99:4477-4482.

3 Mansky KC, Sulzbacher S, Purdom G, et al. The microphthalmia transcription factor and the related helix-loop-helix zipper factors TFE-3 and TFE-C collaborate to activate the tartrate-resistant acid phosphatase promoter. J Leukoc Biol 2002;71:304-310.

4 Mansky KC, Marfatia K, Purdom GH, et al. The microphthalmia transcription factor (MITF) contains two N-terminal domains required for transactivation of osteoclast target promoters and rescue of mi mutant osteoclasts. J Leukoc Biol 2002;71:295-303.

5 Mansky KC, Sankar U, Han J, et al. Microphthalmia transcription factor is a target of the p38 MAPK pathway in response to receptor activator of NF-kappa B ligand signaling. J Biol Chem 2002;277:11077-11083.

6 Boyce BF, Hughes DE, Wright KR, et al. Recent advances in bone biology provide insight into the pathogenesis of bone diseases. Lab Invest 1999;79: 83-94.

7 Motyckova G, Weilbaecher KN, Horstmann M, et al. Linking osteopetrosis and pycnodysostosis: regulation of cathepsin $\mathrm{K}$ expression by the microphthalmia transcription factor family. Proc Natl Acad Sci USA 2001;98:5798-5803.

8 Luchin A, Purdom G, Murphy K, et al. The microphthalmia transcription factor regulates expression of the tartrate-resistant acid phosphatase gene during terminal differentiation of osteoclasts. J Bone Miner Res 2000;15:451-460.

9 Kawaguchi N, Noda M. Mitf is expressed in osteoclast progenitors in vitro. Exp Cell Res 2000;260:284-291.

10 Vignery A, Raymond MJ, Qian HY, et al. Multinucleated rat alveolar macrophages express functional receptors for calcitonin. Am J Physiol 1991;261(Pt 2): F1026-F1032.

11 Vignery A. Osteoclasts and giant cells: macrophagemacrophage fusion mechanism. Int J Exp Pathol 2000;81:291-304.

12 Jimi E, Nakamura I, Duong LT, et al. Interleukin 1 induces multinucleation and bone-resorbing activity of osteoclasts in the absence of osteoblasts/stromal cells. Exp Cell Res 1999;247:84-93.

13 Rolauffs B, Bernhardt TM, Von Eiff C, et al. Osteopetrosis, femoral fracture, and chronic osteomyelitis caused by Staphylococcus aureus small colony variants (SCV) treated by girdlestone resection-6-year follow-up. Arch Orthop Trauma Surg 2002;122: 547-550.

14 Kempson RLF, Fletcher CDM, Evans HL, et al. Synovial tumors. In: Rosai J (ed). Tumors of the Soft Tissue, Atlas of tumor Pathology Third Series; Vol. 30, 3rd edn. Armed Forces Institute of Pathology: Washington, DC, 2001, pp 387-394.

15 Komiya S, et al. Characterization of cells cultured from human giant-cell tumors of bone. Phenotypic relationship to the monocyte-macrophage and osteoclast. Clin Orthop 1990;258:304-309.

16 Wuelling M, Engels C, Jesse N, et al. Histogenesis of giant cell tumors. Pathologe 2002;23:332-339.

17 Zheng $\mathrm{MH}$, Robbins $\mathrm{P}, \mathrm{Xu}$ J, et al. The histogenesis of giant cell tumour of bone: a model of interaction between neoplastic cells and osteoclasts. Histol Histopathol 2001;16:297-307.

18 Weiss SW, Goldblum JR. Enzinger and Weiss's Soft Tissue Tumors, 4th edn. Mosby: St Louis, 2001. 